\title{
Primal-dual subgradient method for constrained convex optimization problems
}

\author{
Michael R. Metel • Akiko Takeda
}

Received: date / Accepted: date

\begin{abstract}
This paper considers a general convex constrained problem setting where functions are not assumed to be differentiable nor Lipschitz continuous. Our motivation is in finding a simple first-order method for solving a wide range of convex optimization problems with minimal requirements. We study the method of weighted dual averages [7] in this setting and prove that it is an optimal method.
\end{abstract}

Keywords convex optimization · subgradient method · non-smooth optimization · iteration complexity $\cdot$ constrained optimization

\section{Declarations}

Funding: The research of the first author is supported in part by JSPS KAKENHI Grants No.19H04069. The research of the second author is supported in part by JSPS KAKENHI Grants No. $17 \mathrm{H} 01699$ and 19H04069.

Conflicts of interest/competing interests, availability of data and material, and code availability: Not applicable.

Michael R. Metel

RIKEN Center for Advanced Intelligence Project

Tokyo, Japan

E-mail: michaelros.metel@riken.jp

Akiko Takeda

Department of Creative Informatics

Graduate School of Information Science and Technology

The University of Tokyo

Tokyo, Japan

RIKEN Center for Advanced Intelligence Project

Tokyo, Japan

E-mail: takeda@mist.i.u-tokyo.ac.jp 


\section{Introduction}

In this work we are interested in constrained minimization problems,

$$
\begin{array}{rl}
\min _{x \in \mathbb{R}^{d}} & f(x) \\
\text { s.t. } & f_{i}(x) \leq 0 \quad i=1, \ldots, n \\
& h_{i}(x)=0 \quad i=1, \ldots, p,
\end{array}
$$

where $f(x)$ and all $f_{i}(x)$ are convex functions and all $h_{i}(x)$ are affine functions from $\mathbb{R}^{d}$ to $\mathbb{R}$. Our goal is to develop an algorithm with a proven convergence rate to the constrained minimum of $f(x)$ without any further assumptions besides standard regularity conditions. In particular, we do not assume that $f(x)$ or the functions $\left\{f_{i}(x)\right\}$ are differentiable nor Lipschitz continuous, and we do not assume a priori that algorithm iterates are constrained to any bounded set. All that is required is that an optimal primal and dual solution exist and that strong duality holds.

Convex functions which are neither differentiable nor Lipschitz continuous arise naturally in optimization problems, such as the maximum of a set of quadratic functions and the unconstrained soft-margin SVM formulation 8 , Ch. 15]. Our work's main motivation though is in finding a general algorithm which can be applied to a wide range of applications without the need for detailed function properties or problem specific parameter tuning.

In terms of non-asymptotic convergence guarantees for constrained nonsmooth convex optimization problems, there are deterministic algorithms, such as the subgradient method [6, Theorem 3.2.3] and its extension using mirror descent [1], as well as algorithms for stochastic optimization settings such as the cooperative stochastic approximation algorithm [5], which can be seen as a stochastic extension of the subgradient method, and the primal-dual stochastic gradient method 9, which is based on the analysis of the augmented Lagrangian. After $K$ iterations, all of the algorithms discussed have a proven rate of convergence of $O\left(\frac{1}{\sqrt{K}}\right)$ towards an optimal solution, which is the best rate achievable using a first-order method, in the sense of matching the lower complexity bound for the unconstrained version of our problem setting [6. Section 3.2.1]. All of these algorithms' convergence results rely on some combination of a compact feasible region, bounds on the subgradients, or bounds on the constraint functions though.

If we consider the unconstrained problem with $n=p=0$, recent works include [3], which proved that the convergence rate of the subgradient method holds under the more relaxed assumption compared to Lipschitz continuity, that $f(x)-f\left(x^{*}\right) \leq D\left(\left\|x-x^{*}\right\|_{2}\right)$ holds where $x^{*}$ is an optimal solution and $D(\cdot)$ is a non-negative non-decreasing function. The convergence rate of $O\left(\frac{1}{\sqrt{K}}\right)$ for the general unconstrained convex optimization problem was solved earlier though in [7, with the method of weighted dual averages. The iterates of the algorithm can be shown to be bounded for a range of convex optimization problems, including unconstrained minimization without the assumption of 
a global Lipschitz parameter. The path taken in this paper is to apply the method of weighted dual averages, presented as Algorithm 1, to the general convex constrained problem (1) and establish the same rate of convergence as previous works under our more relaxed assumptions.

\section{Preliminaries}

We define the Lagrangian function as

$$
L(x, \mu, \theta):=f(x)+\sum_{i=1}^{n} \mu_{i} f_{i}(x)+\sum_{i=1}^{p} \theta_{i} h_{i}(x),
$$

and the dual problem as

$$
\max _{\substack{\mu \geq \mathbb{R}_{+}^{n} \\ \theta \in \mathbb{R}^{p}}} \min _{x \in \mathbb{R}^{d}} L(x, \mu, \theta)
$$

The following assumptions are sufficient for (1) to be a convex optimization problem with an optimal primal and dual solution with strong duality.

\section{Assumptions 1}

1. $f(x)$ and $f_{i}(x)$ for $i=1, \ldots, n$ are real-valued convex functions, and $h_{i}(x)$ for $i=1, \ldots, p$ are affine functions, all over $\mathbb{R}^{d}$.

2. Slater's condition holds: there exists an $\hat{x} \in \mathbb{R}^{d}$ such that $f_{i}(\hat{x})<0$ for $i=1, \ldots, n$ and $h_{i}(\hat{x})=0$ for $i=1, \ldots, p$.

3. There exists an optimal solution, denoted $x^{*}$.

These assumptions are sufficient since by the finiteness of $f\left(x^{*}\right)$ and Slater's condition, strong duality holds and there exists at least one dual optimal solution $\left(\mu^{*}, \theta^{*}\right)$ [2, Prop. 5.3.5].

Strong duality holds if and only if $\left(x^{*}, \mu^{*}, \theta^{*}\right)$ is a saddle point of $L(x, \mu, \theta)$ [2, Prop. 3.4.1], i.e. $\forall x \in \mathbb{R}^{d}, \mu \geq \mathbb{R}_{+}^{n}$, and $\theta \in \mathbb{R}^{p}$,

$$
L\left(x^{*}, \mu, \theta\right) \leq L\left(x^{*}, \mu^{*}, \theta^{*}\right) \leq L\left(x, \mu^{*}, \theta^{*}\right) .
$$

We will work with an unconstrained version of (1), written as

$$
\min _{x \in \mathbb{R}^{d}} \max _{\lambda \geq 0} F(x, \lambda):=f(x)+\lambda \bar{f}(x),
$$

where

$$
\bar{f}(x):=\max \left(f_{1}(x), f_{2}(x), \ldots, f_{n}(x),\left|h_{1}(x)\right|,\left|h_{2}(x)\right|, \ldots,\left|h_{p}(x)\right|\right) .
$$

Let $\lambda^{*}=\sum_{i=1}^{n} \mu_{i}^{*}+\sum_{i=1}^{p}\left|\theta_{i}^{*}\right|$. By the fact that $\bar{f}\left(x^{*}\right)=0$ and complementary slackness, $\sum_{i=1}^{\bar{n}} \mu_{i}^{*} f_{i}\left(x^{*}\right)=0$,

$$
F\left(x^{*}, \lambda\right)=f\left(x^{*}\right)=L\left(x^{*}, \mu^{*}, \theta^{*}\right) \forall \lambda,
$$


and

$$
\begin{aligned}
L\left(x, \mu^{*}, \theta^{*}\right) & =f(x)+\sum_{i=1}^{n} \mu_{i}^{*} f_{i}(x)+\sum_{i=1}^{p} \theta_{i}^{*} h_{i}(x) \\
& \leq f(x)+\sum_{i=1}^{n} \mu_{i}^{*} f_{i}(x)+\sum_{i=1}^{p}\left|\theta_{i}^{*}\right|\left|h_{i}(x)\right| \\
& \leq f(x)+\lambda^{*} \bar{f}(x) \\
& =F\left(x, \lambda^{*}\right)
\end{aligned}
$$

hence from (2), for all $(x, \lambda) \in \mathbb{R}^{d+1}$,

$$
F\left(x^{*}, \lambda\right) \leq F\left(x, \lambda^{*}\right) .
$$

We will use the following notation for the subgradients needed of $F(x, \lambda)$,

$$
\begin{aligned}
g(x) & \in \partial f(x) \\
g_{i}(x) & \in \partial f_{i}(x) \\
\bar{g}(x) & \in \partial \bar{f}(x)=\operatorname{Conv}\left(\left\{\partial f_{i}(x): f_{i}(x)=\bar{f}(x)\right\} \cup\left\{\partial\left|h_{i}(x)\right|:\left|h_{i}(x)\right|=\bar{f}(x)\right\}\right) \\
G_{x}(x, \lambda) & \in \partial_{x} F(x, \lambda)=\partial f(x)+\lambda \partial \bar{f}(x) \\
G_{\lambda}(x, \lambda) & =\frac{\partial}{\partial \lambda} F(x, \lambda)=\bar{f}(x) \\
G(x, \lambda) & \in \partial F(x, \lambda),
\end{aligned}
$$

where for subdifferential of $\partial \bar{f}(x)$, see for example [6, Lemma 3.1.10]. From a practical perspective, $\bar{g}(x)$ can be taken as an element $\bar{g}(x) \in \partial H(x)$, where $H(x) \in\left\{f_{1}(x), f_{2}(x), \ldots, f_{n}(x),\left|h_{1}(x)\right|,\left|h_{2}(x)\right|, \ldots,\left|h_{p}(x)\right|\right\}$ and $H(x)=\bar{f}(x)$. Following the standard measure of convergence to a primal solution, given an optimal solution $x^{*}$, we define an algorithm's output $\bar{x}$ as an $\left(\epsilon_{1}, \epsilon_{2}\right)$-optimal solution if

$$
f(\bar{x})-f\left(x^{*}\right) \leq \epsilon_{1} \quad \text { and } \quad \bar{f}(\bar{x}) \leq \epsilon_{2}
$$

\section{Weighted dual method}

Convergence to an optimal solution is proven using the method of weighted dual averages of 7] presented as Algorithm1, When convenient we will use the column vector $w:=[x ; \lambda]:=\left[x^{T}, \lambda^{T}\right]^{T}$, and the notation $\bar{G}_{k}:=\frac{\left[G_{x}\left(w_{k}\right) ;-G_{\lambda}\left(w_{k}\right)\right]}{\left\|G\left(w_{k}\right)\right\|_{2}}$ (note that $\left\|\bar{G}_{k}\right\|_{2}=1$ ). 


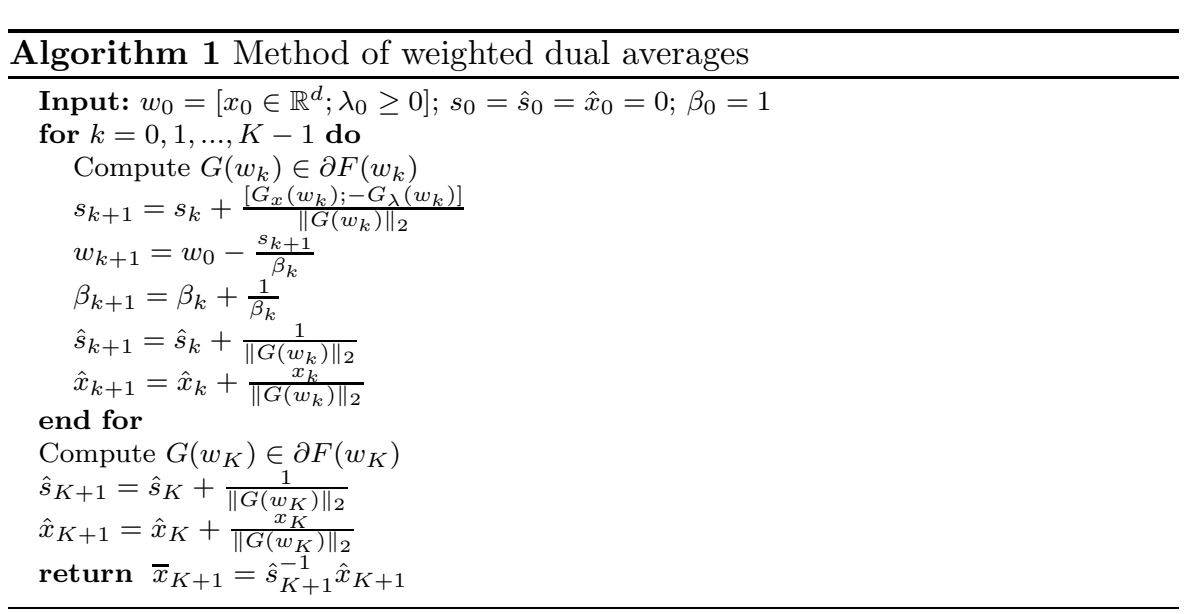

In each iteration $w_{k+1}=w_{0}-\frac{s_{k+1}}{\beta_{k}}$ is the maximizer of

$$
U_{\beta}^{s}(w):=-\left\langle s, w-w_{0}\right\rangle-\frac{\beta}{2}\left\|w-w_{0}\right\|_{2}^{2}
$$

for $s=s_{k+1}$ and $\beta=\beta_{k}$, with

$$
U_{\beta_{k}}^{s_{k+1}}\left(w_{k+1}\right)=\frac{\left\|s_{k+1}\right\|_{2}^{2}}{2 \beta_{k}} .
$$

In addition, $U_{\beta}^{s}(w)$ is strongly concave in $w$ with parameter $\beta$,

$$
U_{\beta}^{s}(w) \leq U_{\beta}^{s}\left(w^{\prime}\right)+\left\langle\nabla U_{\beta}^{s}\left(w^{\prime}\right), w-w^{\prime}\right\rangle-\frac{\beta}{2}\left\|w-w^{\prime}\right\|_{2}^{2} .
$$

Given that $G_{\lambda}\left(w_{k}\right) \geq 0$, it holds that $\lambda_{k+1} \geq \lambda_{k}$, with the $\lambda_{k}$ iterates always remaining feasible. The following property examines the case Algorithm 1 crashes due to $\left\|G\left(w_{k}\right)\right\|_{2}=0$.

Property 1 If $\left\|G\left(w_{k}\right)\right\|_{2}=0$, then $x_{k}$ is an optimal solution of (1).

Proof If $\left\|G\left(w_{k}\right)\right\|_{2}=0$, this implies that $G_{\lambda}\left(w_{k}\right)=\bar{f}\left(x_{k}\right)=0$ and hence $x_{k}$ is a feasible solution. From $\left\|G_{x}\left(w_{k}\right)\right\|_{2}=0,0 \in \partial_{x} F\left(x_{k}, \lambda_{k}\right)$, and as $F\left(x, \lambda_{k}\right)$ is convex in $x, x_{k}$ is a minimizer of $F\left(x, \lambda_{k}\right)$. It follows that for all $x \in \mathbb{R}^{d}$ feasible in (11),

$$
f\left(x_{k}\right)=f\left(x_{k}\right)+\lambda_{k} \bar{f}\left(x_{k}\right) \leq f(x)+\lambda_{k} \bar{f}(x)=f(x) .
$$

A key property of Algorithm 1 is that by redefining $G\left(w_{k}\right)$ appropriately, the iterates are bounded for quite general convex optimization problems. In particular, all that is required is that (11) in the proof below holds for the iterates to be bounded using [7, Theorem 3]. For the sake of completeness we present the full proof for our application in Property 3 . It is convenient to first prove a preliminary property which will be used in Property 3 and Theorem 1 . 
Property 2 For any iterate $\bar{k} \in\{1,2, \ldots, K\}$ of Algorithm 1 , it holds that

$$
\sum_{k=1}^{\bar{k}}\left\langle w_{k}-w_{0}, \bar{G}_{k}\right\rangle \leq-U_{\beta_{\bar{k}}}^{s_{\bar{k}+1}}\left(w_{\bar{k}+1}\right)+\frac{\beta_{\bar{k}}}{2} .
$$

Proof From (5),

$$
\begin{aligned}
U_{\beta_{k}}^{s_{k+1}}\left(w_{k+1}\right) & =\frac{\beta_{k-1}}{\beta_{k}} \frac{\left\|s_{k+1}\right\|_{2}^{2}}{2 \beta_{k-1}} \\
& =\frac{\beta_{k-1}}{\beta_{k}} \frac{\left\|s_{k}+\bar{G}_{k}\right\|_{2}^{2}}{2 \beta_{k-1}} \\
& =\frac{\beta_{k-1}}{\beta_{k}}\left(\frac{\left\|s_{k}\right\|_{2}^{2}}{2 \beta_{k-1}}+\frac{1}{\beta_{k-1}}\left\langle s_{k}, \bar{G}_{k}\right\rangle+\frac{\left\|\bar{G}_{k}\right\|_{2}^{2}}{2 \beta_{k-1}}\right) \\
& =\frac{\beta_{k-1}}{\beta_{k}}\left(U_{\beta_{k-1}}^{s_{k}}\left(w_{k}\right)+\frac{1}{\beta_{k-1}}\left\langle s_{k}, \bar{G}_{k}\right\rangle+\frac{1}{2 \beta_{k-1}}\right) \\
& =\frac{\beta_{k-1}}{\beta_{k}}\left(U_{\beta_{k-1}}^{s_{k}}\left(w_{k}\right)+\left\langle w_{0}-w_{k}, \bar{G}_{k}\right\rangle+\frac{1}{2 \beta_{k-1}}\right) .
\end{aligned}
$$

Rearranging,

$$
\begin{aligned}
\left\langle w_{k}-w_{0}, \bar{G}_{k}\right\rangle & =U_{\beta_{k-1}}^{s_{k}}\left(w_{k}\right)-\frac{\beta_{k}}{\beta_{k-1}} U_{\beta_{k}}^{s_{k+1}}\left(w_{k+1}\right)+\frac{1}{2 \beta_{k-1}} \\
& \leq U_{\beta_{k-1}}^{s_{k}}\left(w_{k}\right)-U_{\beta_{k}}^{s_{k+1}}\left(w_{k+1}\right)+\frac{1}{2 \beta_{k-1}}
\end{aligned}
$$

since $\beta_{k}$ is increasing. Telescoping these inequalities for $k=1, . ., \bar{k}$,

$$
\begin{aligned}
\sum_{k=1}^{\bar{k}}\left\langle w_{k}-w_{0}, \bar{G}_{k}\right\rangle & \leq U_{\beta_{0}}^{s_{1}}\left(w_{1}\right)-U_{\beta_{\bar{k}}}^{s_{\bar{k}+1}}\left(w_{\bar{k}+1}\right)+\sum_{k=1}^{\bar{k}} \frac{1}{2 \beta_{k-1}} \\
& =\frac{1}{2 \beta_{0}}-U_{\beta_{\bar{k}}}^{s_{\bar{k}+1}}\left(w_{\bar{k}+1}\right)+\sum_{k=0}^{\bar{k}-1} \frac{1}{2 \beta_{k}} \\
& =-U_{\beta_{\bar{k}}}^{s_{\bar{k}+1}}\left(w_{\bar{k}+1}\right)+\frac{1}{2}\left(\sum_{k=0}^{\bar{k}-1} \frac{1}{\beta_{k}}+\beta_{0}\right)
\end{aligned}
$$

where $\left\|s_{1}\right\|_{2}=1$ was used in the first equality, and $\beta_{0}=1$ was used in the second equality. Expanding the recursion $\beta_{k}=\frac{1}{\beta_{k}-1}+\beta_{k-1}$,

$$
\sum_{k=1}^{\bar{k}}\left\langle w_{k}-w_{0}, \bar{G}_{k}\right\rangle \leq-U_{\beta_{\bar{k}}}^{s_{\bar{k}+1}}\left(w_{\bar{k}+1}\right)+\frac{\beta_{\bar{k}}}{2} .
$$


Property 3 For any iterate $\bar{k} \in\{1,2, \ldots, K\}$ of Algorithm 1, it holds that

$$
\left\|w_{\bar{k}}-w^{*}\right\|_{2} \leq\left\|w_{0}-w^{*}\right\|_{2}+1
$$

with the inequality being strict when $w_{0} \neq w^{*}$.

Proof Given the convexity of $F(x, \lambda)$ in $x$ and linearity in $\lambda$,

$$
F\left(x^{*}, \lambda_{k}\right) \geq F\left(x_{k}, \lambda_{k}\right)+\left\langle G_{x}\left(x_{k}, \lambda_{k}\right), x^{*}-x_{k}\right\rangle
$$

and

$$
F\left(x_{k}, \lambda^{*}\right)=F\left(x_{k}, \lambda_{k}\right)+\left\langle G_{\lambda}\left(x_{k}, \lambda_{k}\right), \lambda^{*}-\lambda_{k}\right\rangle .
$$

Subtracting (9) from (10) and using (3),

$$
0 \leq\left\langle\left[G_{x}\left(w_{k}\right) ;-G_{\lambda}\left(w_{k}\right)\right], w_{k}-w^{*}\right\rangle .
$$

For the case when $\bar{k}=1$,

$$
\begin{aligned}
\left\|w_{1}-w^{*}\right\|_{2}^{2} & =\left\|w_{0}-w^{*}-\bar{G}_{0}\right\|_{2}^{2} \\
& =\left\|w_{0}-w^{*}\right\|_{2}^{2}-2\left\langle w_{0}-w^{*}, \bar{G}_{0}\right\rangle+1 \\
& \leq\left\|w_{0}-w^{*}\right\|_{2}^{2}+1,
\end{aligned}
$$

where the last line uses (11). When $\bar{k}>1$ from (11),

$$
\begin{aligned}
0 & \leq \sum_{k=1}^{\bar{k}-1}\left\langle w_{k}-w^{*}, \bar{G}_{k}\right\rangle \\
& =\sum_{k=1}^{\bar{k}-1}\left\langle w_{0}-w^{*}, \bar{G}_{k}\right\rangle+\sum_{k=1}^{\bar{k}-1}\left\langle w_{k}-w_{0}, \bar{G}_{k}\right\rangle \\
& \leq \sum_{k=1}^{\bar{k}-1}\left\langle w_{0}-w^{*}, \bar{G}_{k}\right\rangle-U_{\beta_{\bar{k}-1}}^{s_{\bar{k}}}\left(w_{\bar{k}}\right)+\frac{\beta_{\bar{k}-1}}{2} \\
& =\left\langle w_{0}-w^{*}, s_{\bar{k}}\right\rangle-U_{\beta_{\bar{k}-1}}^{s_{\bar{k}}}\left(w_{\bar{k}}\right)+\frac{\beta_{\bar{k}-1}}{2}
\end{aligned}
$$

where the second inequality uses Property 2, and the second equality holds since $s_{k+1}=s_{k}+\bar{G}_{k}$. Considering inequality (6) with $s=s_{\bar{k}}, \beta=\beta_{\bar{k}-1}$, $w=w^{*}$, and $w^{\prime}=w_{\bar{k}}$,

$$
\begin{aligned}
U_{\beta_{\bar{k}-1}}^{s_{\bar{k}}}\left(w^{*}\right) & \leq U_{\beta_{\bar{k}-1}}^{s_{\bar{k}}}\left(w_{\bar{k}}\right)+\left\langle\nabla U_{\beta_{\bar{k}-1}}^{s_{\bar{k}}}\left(w_{\bar{k}}\right), w^{*}-w_{\bar{k}}\right\rangle-\frac{\beta_{\bar{k}-1}}{2}\left\|w^{*}-w_{\bar{k}}\right\|_{2}^{2} \\
& =U_{\beta_{\bar{k}-1}}^{s_{\bar{k}}}\left(w_{\bar{k}}\right)-\frac{\beta_{\bar{k}-1}}{2}\left\|w^{*}-w_{\bar{k}}\right\|_{2}^{2},
\end{aligned}
$$


given that $w_{\bar{k}}$ is the maximum of $U_{\beta_{\bar{k}-1}}^{s_{\bar{k}}}(w)$. Applying this inequality in (13),

$$
\begin{aligned}
0 & \leq\left\langle w_{0}-w^{*}, s_{\bar{k}}\right\rangle-U_{\beta_{\bar{k}-1}}^{s_{\bar{k}}}\left(w^{*}\right)-\frac{\beta_{\bar{k}-1}}{2}\left\|w^{*}-w_{\bar{k}}\right\|_{2}^{2}+\frac{\beta_{\bar{k}-1}}{2} \\
& =\left\langle w_{0}-w^{*}, s_{\bar{k}}\right\rangle+\left\langle s_{\bar{k}}, w^{*}-w_{0}\right\rangle+\frac{\beta_{\bar{k}-1}}{2}\left\|w^{*}-w_{0}\right\|_{2}^{2}-\frac{\beta_{\bar{k}-1}}{2}\left\|w^{*}-w_{\bar{k}}\right\|_{2}^{2}+\frac{\beta_{\bar{k}-1}}{2} \\
& =\frac{\beta_{\bar{k}-1}}{2}\left\|w^{*}-w_{0}\right\|_{2}^{2}-\frac{\beta_{\bar{k}-1}}{2}\left\|w^{*}-w_{\bar{k}}\right\|_{2}^{2}+\frac{\beta_{\bar{k}-1}}{2}
\end{aligned}
$$

where the first equality uses the definition of $U_{\beta_{\bar{k}-1}}^{s_{\bar{k}}}\left(w^{*}\right)$ (44). Rearranging,

$$
\left\|w^{*}-w_{\bar{k}}\right\|_{2}^{2} \leq\left\|w^{*}-w_{0}\right\|_{2}^{2}+1
$$

Now for all $\bar{k}$, from (12) and (14),

$$
\begin{aligned}
\left(\left\|w^{*}-w_{0}\right\|_{2}+1\right)^{2} & =\left\|w^{*}-w_{0}\right\|_{2}^{2}+2\left\|w^{*}-w_{0}\right\|+1 \\
& \geq\left\|w^{*}-w_{\bar{k}}\right\|_{2}^{2}+2\left\|w^{*}-w_{0}\right\|,
\end{aligned}
$$

so that

$$
\left\|w^{*}-w_{0}\right\|_{2}+1 \geq\left\|w^{*}-w_{\bar{k}}\right\|_{2},
$$

with (15) being strict when $w^{*} \neq w_{0}$.

In order to prove the convergence result of Algorithm 1, we require bounding the norm of the subgradients $G\left(w_{k}\right)$.

Property 4 There exists a constant $L$ such that $\left\|g\left(x_{k}\right)\right\|_{2} \leq L,\left\|\bar{g}\left(x_{k}\right)\right\|_{2} \leq L$, $\bar{f}\left(x_{k}\right) \leq L\left\|x_{k}-x^{*}\right\|_{2}$, and

$$
\left\|G\left(w_{k}\right)\right\|_{2} \leq L\left(2\left\|w_{0}-w^{*}\right\|_{2}+\lambda^{*}+3\right)
$$

for all $k$.

Proof Recall that $g(x) \in \partial f(x)$ and $\bar{g}(x) \in \partial \bar{f}(x)$,

$$
\begin{aligned}
\left\|G\left(w_{k}\right)\right\|_{2} & =\left\|\left[G_{x}\left(w_{k}\right) ; G_{\lambda}\left(w_{k}\right)\right]\right\|_{2} \\
& =\left\|\left[g\left(x_{k}\right)+\lambda_{k} \bar{g}\left(x_{k}\right) ; \bar{f}\left(x_{k}\right)\right]\right\|_{2} \\
& \leq\left\|g\left(x_{k}\right)\right\|_{2}+\lambda_{k}\left\|\bar{g}\left(x_{k}\right)\right\|_{2}+\bar{f}\left(x_{k}\right) .
\end{aligned}
$$

Property 3 ensures that the iterates of Algorithm 1 are bounded in a convex compact region, $w_{k} \in D:=\left\{w:\left\|w-w^{*}\right\|_{2} \leq\left\|w_{0}-w^{*}\right\|_{2}+1\right\}$. This implies that $x_{k} \in D_{x}:=\left\{x:\left\|x-x^{*}\right\|_{2} \leq\left\|w_{0}-w^{*}\right\|_{2}+1\right\}$ and $\lambda_{k} \in D_{\lambda}:=\{\lambda:$ $\left.\left|\lambda-\lambda^{*}\right| \leq\left\|w_{0}-w^{*}\right\|_{2}+1\right\}$. It follows that there exists an $L_{1} \geq 0$ such that $f(x)$ is $L_{1}$-Lipschitz continuous on $D_{x}$ [4, Theorem IV.3.1.2],

$$
\left|f(x)-f\left(x^{\prime}\right)\right| \leq L_{1}\left\|x-x^{\prime}\right\|_{2},
$$


for all $x, x^{\prime} \in D_{x}$. Assuming that $w_{0} \neq w^{*}, x_{k} \in \operatorname{Int} D_{x}$. For any $x \in \operatorname{Int} D_{x}$, taking $\theta>0$ small enough such that $x^{\prime}=x+\theta \frac{g(x)}{\|g(x)\|_{2}} \in D_{x}$,

$$
\begin{array}{rlrl}
\left\langle g(x), x^{\prime}-x\right\rangle & \leq f\left(x^{\prime}\right)-f(x) \\
\Longrightarrow & \left\langle g(x), x^{\prime}-x\right\rangle & \leq L_{1}\left\|x^{\prime}-x\right\|_{2} \\
\Longrightarrow & \left\langle g(x), \theta \frac{g(x)}{\|g(x)\|_{2}}\right\rangle & \leq L_{1} \theta \\
& \Rightarrow & \|g(x)\|_{2} & \leq L_{1} .
\end{array}
$$

If $w_{0}=w^{*}, x_{k} \in \operatorname{Int} D_{x}^{\delta}:=\left\{x:\left\|x-x^{*}\right\|_{2} \leq \delta+1\right\}$ for any $\delta>0$, and $L_{1}$ can be increased such that (17) holds over $D_{x}^{\delta}$ so that (18) holds for all $x \in D_{x}$. Similarly, there exists an $L_{2} \geq 0$ such that $\left|\bar{f}(x)-\bar{f}\left(x^{\prime}\right)\right| \leq L_{2}\left\|x-x^{\prime}\right\|_{2}$ and $\|\bar{g}(x)\|_{2} \leq L_{2}$ for all $x, x^{\prime} \in D_{x}$. In addition,

$$
\begin{aligned}
\bar{f}\left(x_{k}\right) & =\left|\bar{f}\left(x_{k}\right)-\bar{f}\left(x^{*}\right)\right| \\
& \leq L_{2}\left\|x_{k}-x^{*}\right\|_{2} \\
& \leq L_{2}\left(\left\|w_{0}-w^{*}\right\|_{2}+1\right),
\end{aligned}
$$

and $\lambda_{k} \leq\left\|w_{0}-w^{*}\right\|_{2}+1+\lambda^{*}$ from the definition of $D_{\lambda}$. Combining these bounds in (16) and taking $L=\max \left(L_{1}, L_{2}\right)$,

$$
\begin{aligned}
\left\|G\left(w_{k}\right)\right\|_{2} & \leq\left\|g\left(x_{k}\right)\right\|_{2}+\lambda_{k}\left\|\bar{g}\left(x_{k}\right)\right\|_{2}+\bar{f}\left(x_{k}\right) \\
& \leq L_{1}+\left(\left\|w_{0}-w^{*}\right\|_{2}+1+\lambda^{*}\right) L_{2}+L_{2}\left(\left\|w_{0}-w^{*}\right\|_{2}+1\right) \\
& \leq L\left(2\left\|w_{0}-w^{*}\right\|_{2}+\lambda^{*}+3\right) .
\end{aligned}
$$

For all $k$ the value of $\beta_{k}$ can be bounded as follows using induction.

Property 5 [7, Lemma 3]

$$
\beta_{k} \leq \frac{1}{1+\sqrt{3}}+\sqrt{2 k+1}
$$

We can now prove a convergence rate of $O\left(\frac{1}{\sqrt{K}}\right)$ to an optimal solution of problem (11). We will define the bound on $\left\|G\left(w_{k}\right)\right\|_{2}$ from Property (4) as $C:=L\left(2\left\|w_{0}-w^{*}\right\|_{2}+\lambda^{*}+3\right)$.

Theorem 1 Running Algorithm 1 for $K$ iterations,

$$
f\left(\bar{x}_{K+1}\right)-f\left(x^{*}\right) \leq \frac{C\left(\left\|w_{0}-w^{*}\right\|_{2}^{2}+1\right)}{2(K+1)}\left(\frac{1}{1+\sqrt{3}}+\sqrt{2 K+1}\right)
$$

and

$$
\bar{f}\left(\bar{x}_{K+1}\right) \leq \frac{C\left(4\left(\left\|w_{0}-w^{*}\right\|_{2}+1\right)^{2}+1\right)}{2(K+1)}\left(\frac{1}{1+\sqrt{3}}+\sqrt{2 K+1}\right),
$$

where $C:=L\left(2\left\|w_{0}-w^{*}\right\|_{2}+\lambda^{*}+3\right)$. 
Proof Applying Property 2 with $\bar{k}=K$, and recalling that $w_{K+1}$ maximizes $U_{\beta_{K}}^{s_{K+1}}(w)$ (4),

$$
\begin{aligned}
\frac{\beta_{K}}{2} & \geq \sum_{k=1}^{K}\left\langle w_{k}-w_{0}, \bar{G}_{k}\right\rangle+U_{\beta_{K}}^{s_{K+1}}\left(w_{K+1}\right) \\
& =\sum_{k=1}^{K}\left\langle w_{k}-w_{0}, \bar{G}_{k}\right\rangle+\max _{w \in \mathbb{R}^{d+1}}\left\{-\left\langle\sum_{k=0}^{K} \bar{G}_{k}, w-w_{0}\right\rangle-\frac{\beta_{K}}{2}\left\|w-w_{0}\right\|_{2}^{2}\right\} \\
& =\max _{w \in \mathbb{R}^{d+1}}\left\{\sum_{k=0}^{K}-\left\langle\bar{G}_{k}, w-w_{k}\right\rangle-\frac{\beta_{K}}{2}\left\|w-w_{0}\right\|_{2}^{2}\right\} .
\end{aligned}
$$

Like $\bar{x}_{K+1}$, let $\bar{w}_{K+1}:=\hat{s}_{K+1}^{-1} \sum_{k=0}^{K} \frac{w_{k}}{\left\|G\left(w_{k}\right)\right\|_{2}}$ and $\bar{\lambda}_{K+1}:=\hat{s}_{K+1}^{-1} \sum_{k=0}^{K} \frac{\lambda_{k}}{\left\|G\left(w_{k}\right)\right\|_{2}}$. Multiplying both sides of (19) by $\hat{s}_{K+1}^{-1}$,

$$
\begin{aligned}
\hat{s}_{K+1}^{-1} \frac{\beta_{K}}{2} & \geq \hat{s}_{K+1}^{-1} \max _{w \in \mathbb{R}^{d+1}}\left\{\sum_{k=0}^{K}-\left\langle\bar{G}_{k}, w-w_{k}\right\rangle-\frac{\beta_{K}}{2}\left\|w-w_{0}\right\|_{2}^{2}\right\} \\
& =\hat{s}_{K+1}^{-1} \max _{w \in \mathbb{R}^{d+1}}\left\{\sum_{k=0}^{K}-\left\langle\frac{G_{x}\left(w_{k}\right)}{\left\|G\left(w_{k}\right)\right\|_{2}}, x-x_{k}\right\rangle+\left\langle\frac{G_{\lambda}\left(w_{k}\right)}{\left\|G\left(w_{k}\right)\right\|_{2}}, \lambda-\lambda_{k}\right\rangle-\frac{\beta_{K}}{2}\left\|w-w_{0}\right\|_{2}^{2}\right\} \\
& \geq \hat{s}_{K+1}^{-1} \max _{w \in \mathbb{R}^{d+1}}\left\{\sum_{k=0}^{K} \frac{F\left(x_{k}, \lambda_{k}\right)-F\left(x, \lambda_{k}\right)}{\left\|G\left(w_{k}\right)\right\|_{2}}+\frac{F\left(x_{k}, \lambda\right)-F\left(x_{k}, \lambda_{k}\right)}{\left\|G\left(w_{k}\right)\right\|_{2}}-\frac{\beta_{K}}{2}\left\|w-w_{0}\right\|_{2}^{2}\right\} \\
& =\hat{s}_{K+1}^{-1} \max _{w \in \mathbb{R}^{d+1}}\left\{\sum_{k=0}^{K} \frac{F\left(x_{k}, \lambda\right)-F\left(x, \lambda_{k}\right)}{\left\|G\left(w_{k}\right)\right\|_{2}}-\frac{\beta_{K}}{2}\left\|w-w_{0}\right\|_{2}^{2}\right\} \\
& =\max _{w \in \mathbb{R}^{d+1}}\left\{\hat{s}_{K+1}^{-1} \sum_{k=0}^{K} \frac{F\left(x_{k}, \lambda\right)-F\left(x, \lambda_{k}\right)}{\left\|G\left(w_{k}\right)\right\|_{2}}-\hat{s}_{K+1}^{-1} \frac{\beta_{K}}{2}\left\|w-w_{0}\right\|_{2}^{2}\right\} \\
& \geq \max _{w \in \mathbb{R}^{d+1}}\left\{F\left(\bar{x}_{K+1}, \lambda\right)-F\left(x, \bar{\lambda}_{K+1}\right)-\hat{s}_{K+1}^{-1} \frac{\beta_{K}}{2}\left\|w-w_{0}\right\|_{2}^{2}\right\} \\
& =\max _{w \in \mathbb{R}^{d+1}}\left\{f\left(\bar{x}_{K+1}\right)+\lambda \bar{f}\left(\bar{x}_{K+1}\right)-f(x)-\bar{\lambda}_{K+1} \bar{f}(x)-\hat{s}_{K+1}^{-1} \frac{\beta_{K}}{2}\left\|w-w_{0}\right\|_{2}^{2}\right\},
\end{aligned}
$$

where the second inequality follows from the convexity and linearity of $F(x, \lambda)$ in $x$ and $\lambda$, respectively. The third inequality uses Jensen's inequality and linearity: $\bar{x}_{K+1}=\hat{s}_{K+1}^{-1} \sum_{k=0}^{K} \frac{x_{K}}{\left\|G\left(w_{k}\right)\right\|_{2}}$ is a convex combination of $\left\{x_{k}\right\}$, so by Jensen's inequality,

$$
F\left(\bar{x}_{K+1}, \lambda\right) \leq \hat{s}_{K+1}^{-1} \sum_{k=0}^{K} \frac{F\left(x_{k}, \lambda\right)}{\left\|G\left(w_{k}\right)\right\|_{2}},
$$


and $\bar{\lambda}_{K+1}=\hat{s}_{K+1}^{-1} \sum_{k=0}^{K} \frac{\lambda_{k}}{\left\|G\left(w_{k}\right)\right\|_{2}}$, so by the linearity of of $F(x, \lambda)$ in $\lambda$,

$$
\hat{s}_{K+1}^{-1} \sum_{k=0}^{K} \frac{-F\left(x, \lambda_{k}\right)}{\left\|G\left(w_{k}\right)\right\|_{2}}=-F\left(x, \bar{\lambda}_{K+1}\right) .
$$

Given the maximum function, the inequality (20) holds for any choice of $w$. We consider two cases, the first being $x=\bar{x}_{K+1}$ and $\lambda=\bar{\lambda}_{K+1}+1$. From (20),

$$
\begin{aligned}
\hat{s}_{K+1}^{-1} \frac{\beta_{K}}{2} \geq & f\left(\bar{x}_{K+1}\right)+\left(\bar{\lambda}_{K+1}+1\right) \bar{f}\left(\bar{x}_{K+1}\right)-f\left(\bar{x}_{K+1}\right)-\bar{\lambda}_{K+1} \bar{f}\left(\bar{x}_{K+1}\right) \\
& -\hat{s}_{K+1}^{-1} \frac{\beta_{K}}{2}\left\|\left[\bar{x}_{K+1} ; \bar{\lambda}_{K+1}+1\right]-w_{0}\right\|_{2}^{2} \\
= & \bar{f}\left(\bar{x}_{K+1}\right)-\hat{s}_{K+1}^{-1} \frac{\beta_{K}}{2}\left\|\left[\bar{x}_{K+1} ; \bar{\lambda}_{K+1}+1\right]-w_{0}\right\|_{2}^{2}
\end{aligned}
$$

Further,

$$
\begin{aligned}
\left\|\left[\bar{x}_{K+1} ; \bar{\lambda}_{K+1}+1\right]-w_{0}\right\|_{2} & \leq\left\|\bar{w}_{K+1}-w_{0}\right\|_{2}+1 \\
& =\left\|\bar{w}_{K+1}-w^{*}+w^{*}-w_{0}\right\|_{2}+1 \\
& \leq\left\|\bar{w}_{K+1}-w^{*}\right\|_{2}+\left\|w^{*}-w_{0}\right\|_{2}+1 \\
& \leq \hat{s}_{K+1}^{-1} \sum_{k=0}^{K} \frac{\left\|w_{k}-w^{*}\right\|_{2}}{\left\|G\left(w_{k}\right)\right\|_{2}}+\left\|w^{*}-w_{0}\right\|_{2}+1 \\
& \leq \hat{s}_{K+1}^{-1} \sum_{k=0}^{K} \frac{\left\|w_{0}-w^{*}\right\|_{2}+1}{\left\|G\left(w_{k}\right)\right\|_{2}}+\left\|w^{*}-w_{0}\right\|_{2}+1 \\
& =2\left(\left\|w_{0}-w^{*}\right\|_{2}+1\right),
\end{aligned}
$$

where the third inequality uses Jensen's inequality: $\bar{w}_{K+1}=\hat{s}_{K+1}^{-1} \sum_{k=0}^{K} \frac{w_{k}}{\left\|G\left(w_{k}\right)\right\|_{2}}$ is a convex combination of $\left\{w_{k}\right\}$. The fourth inequality uses Property 3 . Combining (21) and (22),

$$
\bar{f}\left(\bar{x}_{K+1}\right) \leq \hat{s}_{K+1}^{-1} \frac{\beta_{K}}{2}\left(4\left(\left\|w_{0}-w^{*}\right\|_{2}+1\right)^{2}+1\right) .
$$

The second case will use $w=w^{*}$. Starting from (20),

$$
\begin{aligned}
\hat{s}_{K+1}^{-1} \frac{\beta_{K}}{2} & \geq f\left(\bar{x}_{K+1}\right)+\lambda^{*} \bar{f}\left(\bar{x}_{K+1}\right)-f\left(x^{*}\right)-\bar{\lambda}_{K+1} \bar{f}\left(x^{*}\right)-\hat{s}_{K+1}^{-1} \frac{\beta_{K}}{2}\left\|w^{*}-w_{0}\right\|_{2}^{2} \\
& \geq f\left(\bar{x}_{K+1}\right)-f\left(x^{*}\right)-\hat{s}_{K+1}^{-1} \frac{\beta_{K}}{2}\left\|w^{*}-w_{0}\right\|_{2}^{2},
\end{aligned}
$$

since $\bar{f}\left(x^{*}\right)=0$ and $\lambda^{*} \bar{f}\left(\bar{x}_{K+1}\right) \geq 0$. Rearranging,

$$
f\left(\bar{x}_{K+1}\right)-f\left(x^{*}\right) \leq \hat{s}_{K+1}^{-1} \frac{\beta_{K}}{2}\left(\left\|w_{0}-w^{*}\right\|_{2}^{2}+1\right) .
$$


Using Properties 4 and [5, $\hat{s}_{K+1}^{-1} \frac{\beta_{K}}{2}$ can be bounded as follows.

$$
\begin{aligned}
\hat{s}_{K+1}^{-1} \frac{\beta_{K}}{2} & \leq \frac{1}{2}\left(\sum_{k=0}^{K} \frac{1}{\left\|G\left(w_{k}\right)\right\|_{2}}\right)^{-1}\left(\frac{1}{1+\sqrt{3}}+\sqrt{2 k+1}\right) \\
& \leq \frac{1}{2}\left(\sum_{k=0}^{K} \frac{1}{C}\right)^{-1}\left(\frac{1}{1+\sqrt{3}}+\sqrt{2 k+1}\right) \\
& =\frac{C}{2(K+1)}\left(\frac{1}{1+\sqrt{3}}+\sqrt{2 k+1}\right) .
\end{aligned}
$$

Algorithm 1 is an optimal method as its convergence rate of $O\left(\frac{1}{\sqrt{K}}\right)$ from Theorem 1 matches the lower complexity bound for minimizing the unconstrained version of (11) as discussed in the introduction. The following corollary establishes the $O\left(\min \left(\epsilon_{1}, \epsilon_{2}\right)^{-2}\right)$ iteration complexity required to achieve an $\left(\epsilon_{1}, \epsilon_{2}\right)$-optimal solution.

Corollary 1 An $\left(\epsilon_{1}, \epsilon_{2}\right)$ optimal solution is obtained after running Algorithm 1 for

$$
K \geq \alpha \max \left(\frac{C_{1}}{\epsilon_{1}}, \frac{C_{2}}{\epsilon_{2}}\right)^{2}
$$

iterations, where $C_{1}=C\left(\left\|w_{0}-w^{*}\right\|_{2}^{2}+1\right), C_{2}=C\left(4\left(\left\|w_{0}-w^{*}\right\|_{2}+1\right)^{2}+1\right)$, and $\alpha=\frac{1}{2}\left(\frac{1}{\sqrt{8}(1+\sqrt{3})}+1\right)^{2}$.

Proof From Theorem 1 for $i=1,2$, we need to compute a lower bound on $K$ which ensures that

$$
\frac{C_{i}}{2(K+1)}\left(\frac{1}{1+\sqrt{3}}+\sqrt{2 K+1}\right) \leq \epsilon_{i} .
$$

Since for $K \geq 1$,

$$
\begin{aligned}
& \frac{\sqrt{K}}{2(K+1)}\left(\frac{1}{1+\sqrt{3}}+\sqrt{2 K+1}\right) \\
= & \frac{\sqrt{K}}{2(1+\sqrt{3})(K+1)}+\frac{\sqrt{2 K^{2}+K}}{2(K+1)} \\
< & \frac{1}{4(1+\sqrt{3})}+\frac{1}{\sqrt{2}} \\
= & \frac{1}{\sqrt{2}}\left(\frac{1}{\sqrt{8}(1+\sqrt{3})}+1\right) \\
= & \sqrt{\alpha},
\end{aligned}
$$

it holds that

$$
\frac{C_{i}}{2(K+1)}\left(\frac{1}{1+\sqrt{3}}+\sqrt{2 K+1}\right) \leq \frac{\sqrt{\alpha} C_{i}}{\sqrt{K}} .
$$


To ensure convergence within $\epsilon_{i}$, it is sufficient for $\epsilon_{i} \geq \frac{\sqrt{\alpha} C_{i}}{\sqrt{K}}$, or that $K \geq$ $\alpha\left(\frac{C_{i}}{\epsilon_{i}}\right)^{2}$. Taking the maximum over $i$ gives the result.

\section{Conclusion}

In this paper we have established the existence of a simple first-order method for the general convex constrained optimization problem (1) without the need for differentiability nor Lipschitz continuity. We see this as a general use algorithm for practitioners since it requires minimal knowledge of the problem, with no parameter tuning for its implementation, while still achieving the optimal convergence rate for first-order methods.

\section{References}

1. Beck, A., Ben-Tal, A., Guttmann-Beck, N., Tetruashvili, L.: The CoMirror algorithm for solving nonsmooth constrained convex problems. Operations Research Letters 38(6), 493-498 (2010)

2. Bertsekas, D.P.: Convex Optimization Theory. Athena Scientific (2009)

3. Grimmer, B.: Convergence Rates for Deterministic and Stochastic Subgradient Methods without Lipschitz Continuity. SIAM Journal on Optimization 29(2), 1350-1365 (2019)

4. Hiriart-Urruty, J.B., Lemaréchal, C.: Convex Analysis and Minimization Algorithms I: Fundamentals. Springer-Verlag (1996)

5. Lan, G., Zhou, Z.: Algorithms for stochastic optimization with functional or expectation constraints. Computational Optimization and Applications 76, 461-498 (2020)

6. Nesterov, Y.: Introductory Lectures on Convex Optimization: A Basic Course. Springer Science+Business Media (2004)

7. Nesterov, Y.: Primal-dual subgradient methods for convex problems. Mathematical Programming 120(1), 221-259 (2009)

8. Shalev-Shwartz, S., Ben-David, S.: Understanding Machine Learning: From Theory to Algorithms. Cambridge University Press (2014)

9. Xu, Y.: Primal-Dual Stochastic Gradient Method for Convex Programs with Many Functional Constraints. SIAM Journal on Optimization 30(2), 1664-1692 (2020) 